\title{
Osteonecrose dos Maxilares associada ao uso crônico de bisfosfonatos: relato de caso
}

\author{
Jaw osteonecrosis associated with chronic bisphosphonates use: case report
}

Osteonecrosis de la mandíbula asociada con el uso crónico de bisfosfonatos: reporte de caso

Wanderley Barros dos Santos ${ }^{1 *}$, Renata da Silva Pereira ${ }^{1}$, Vanessa Cristina de Branco Gonçalves ${ }^{2}$, Suellen Vieira do Nascimento ${ }^{3}$, Alberto Henrique de Moura Silva ${ }^{4}$, Ana Maria Guerra Costa ${ }^{4}$, Mayã Wanderley Vasconcellos Melo ${ }^{4}$, Ivan José Correia Neto ${ }^{5}$, Yuri Kalinin ${ }^{6}$.

\section{RESUMO}

Objetivo: Descrever um caso de clínico de osteonecrose dos maxilares associada ao uso de bifosfonatos, bem como suas características clínicas e conduta adotada. Detalhamentos de Caso: Paciente do sexo feminino, 67 anos, compareceu ao serviço de Estomatologia devido à presença de lesão em maxila. $\mathrm{Na}$ história médica relatou ser diabética e hipertensa, além de fazer o uso de tratamento para osteoporose há 4 anos, com Alendronato de Sódio. Ao exame físico, foram observadas áreas necróticas com extensa exposição óssea em rebordo maxilar. Inicialmente foi proposto tratamento conservador, com bochechos diários com Clorexidina $0,12 \%$ e acompanhamento mensal, pois a paciente não relatou sintomatologia e não apresentou sequestro ósseo. No entanto, cinco meses após, o osso encontrou-se sequestrado, sendo então necessária a realização da intervenção cirúrgica. Considerações finais: A osteonecrose dos maxilares pode ocorrer devido ao uso prolongado de bifosfonatos. É de fundamental importância o correto diagnóstico pelo cirurgiãodentista, reforçando a orientação àqueles pacientes submetidos ao tratamento com essa classe de medicamentos, visando à prevenção da doença.

Palavras-chave: Bifosfonatos, Osteonecrose, Osteoporose.

\section{ABSTRACT}

Objective: To describe a case of clinician of jaw osteonecrosis associated with the use of bisphosphonates, as well as its clinical characteristics and adopted approach. Case Details: A 67-year-old female patient attended the Stomatology service due to the presence of a maxillary lesion. In medical history reported being diabetic and hypertensive, and have been using treatment for osteoporosis for 4 years with Alendronate Sodium. On physical examination, necrotic areas with extensive bone exposure in the maxillary rim were observed. Conservative treatment was initially proposed, with daily mouthwash with $0.12 \%$ chlorhexidine and monthly follow-up, as the patient did not report symptoms and did not present bone sequestration. However, five months later, the bone was sequestered, so surgical intervention was necessary. Final Considerations: Jaw osteonecrosis may occur due to prolonged use of bisphosphonates. The correct diagnosis by the dentist is of fundamental importance, reinforcing the orientation to those patients submitted to treatment with this class of drugs, aiming at the prevention of the disease.

Key words: Bisphosphonates, Osteonecrosis, Osteoporosis.

\footnotetext{
${ }^{1}$ Centro Universitário Cesmac, Maceió - Alagoas.

2 Universidade Metropolitana de Santos (UNIMES), Santos - São Paulo.

${ }^{3}$ Universidade Federal de São Paulo (UNIFESP), São Paulo.

${ }^{4}$ Faculdade de Tecnologia de Alagoas (FAT), Maceió - Alagoas.

${ }^{5}$ Faculdade de Odontologia da Universidade de São Paulo (FOUSP). São Paulo - SP.

${ }^{6}$ Estomatologista do Centro de Especialidades Odontológicas da Prefeitura de Praia Grande, Praia Grande - São Paulo.

*E-mail: wanderley.barros108@gmail.com
} 


\section{RESUMEN}

Objetivo: Describir un caso clínico de osteonecrosis mandibular asociado con el uso de bifosfonatos, así como sus características clínicas y enfoque adoptado. Detalles del caso: una paciente de 67 años llegó al departamento de Estomatología debido a la presencia de una lesión maxilar. Se ha informado que el historial médico es diabético e hipertensivo, y ha estado usando tratamiento para la osteoporosis durante 4 años con alendronato de sodio. En el examen físico, se observaron áreas necróticas con exposición ósea extensa en el borde superior. Inicialmente se propuso un tratamiento conservador, con enjuague bucal diario con clorhexidina al $0,12 \%$ y seguimiento mensual, ya que el paciente no informó síntomas y no presentó secuestro óseo. Sin embargo, cinco meses después, el hueso fue secuestrado, requiriendo intervención quirúrgica. Consideraciones finales: La osteonecrosis de la mandíbula puede ocurrir debido al uso prolongado de bisfosfonatos. El diagnóstico correcto por parte del dentista es de fundamental importancia, ya que refuerza la orientación para los pacientes sometidos a tratamiento con esta clase de medicamentos, con el objetivo de prevenir la enfermedad.

Palabras clave: Bisfosfonatos, Osteonecrosis, Osteoporosis.

\section{INTRODUÇÃO}

Os bisfosfonatos apresentam-se como uma classificação de fármacos antirreabsortivos, análogos do pirofosfato, no qual reduzem o mecanismo de mineralização óssea de forma dose-dependente devido à inibição das vias de recrutamento dos osteoclastos, que são células responsáveis por mediar este processo absortivo, em áreas de reabsorção óssea, acelerando a apoptose osteoclástica, tendo como resultado a diminuição da taxa remodeladora (DIAS DHS, et al., 2016; FILGUEIRA SL, et al., 2019).

A indicação dos bisfosfonatos foi descrita desde a década de 60 e têm sido amplamente utilizados para tratamentos de distúrbios metabólicos esqueléticos como a osteoporose, visando a estabilização da perda óssea e, consequentemente, diminuir o risco de fraturas vertebrais e de ossos longos, doença de Paget, assim como adjuvante terapêutico em doenças oncológicas envolvendo metástases ósseas, hipercalcemia maligna e mieloma múltiplo (LIMA BKS, et al., 2017; COLÉTE JZ, et al., 2019). O Alendronato de sódio e o ácido zoledrônico, administrados via oral e intravenosa respectivamente, são os medicamentos atualmente mais utilizados no tratamento de distúrbios ósseos metabólicos e tumores. Entretanto, apesar dos benefícios destes, houve ocorrência de diversos relatos de osteonecrose dos maxilares relacionados ao uso de bisfosfonatos, após procedimentos odontológicos invasivos, especialmente em exodontias e instalação de implantes dentários (COLÉTE JZ, et al., 2019; SOUSA AS, et al., 2018).

A prevalência de osteonecrose associada ao tratamento com bisfosfonatos é de 0,019\%, ou seja, 1.9 casos para cada 10.000 pacientes. Estima-se que a incidência em tratamentos prolongados com zoledronato, chega a $1 \%$ e quanto ao alendronato, via oral, seja em torno de $0,1 \%$, podendo aumentar para $0,21 \%$ quando este tratamento se estende por mais de 4 anos (SOUSA AS, et al., 2018).

A ocorrência da osteonecrose associada ao uso desses medicamentos estão diretamente relacionadas com a dose, via de administração e a duração do tratamento, sendo de maior incidência em pacientes que fazem o uso administrado por via intravenosa, com aplicação mensal, por um período maior que três anos. Porém, pacientes portadores de osteoporose com uso crônico de bisfosfonatos por via oral podem apresentar o desenvolvimento dessa lesão (JESUS AP, et al., 2019).

A osteonecrose ocorre devido à redução da vascularização, no qual o tecido ósseo perde a capacidade de remodelação, pela deficiência na angiogênese e acaba resultando em uma área necrótica da região em que foi realizada a intervenção. Pode ocorrer devido a intervenções traumáticas e presença de infecções, associado ao uso contínuo de bisfosfonatos e outras classes de medicamentos, como o Desonumab, agente inibidor do RANKL (ligante do receptor ativador do fator nuclear kappa B), molécula responsável pela ativação osteoclástica durante $\mathrm{o}$ processo de reabsorção que surgiu na tentativa de substituir o alendronato e medicações antiangiogênicas (SOUSA AS, et al., 2018; DUARTE LFM, et al., 2015). 
A osteonecrose dos maxilares é uma doença que afeta tanto a maxila quanto a mandíbula, porém, a localização predominante é de $65 \%$ na mandíbula, sendo relatados apenas $26 \%$ de casos na maxila, e em ambos 9\% (DIAS DHS, et al., 2016).

Clinicamente, observam-se áreas de exposição óssea com presença de necrose e secreção purulenta que não sofre reparação rápida, em pacientes que fazem uso contínuo de bisfosfonatos, sem história prévia de radioterapia. Pode haver queixas de dores e dificuldade mastigatória, além de parestesias, levando a uma frequente diminuição da qualidade de vida (SOUSA AS, et al., 2018; JESUS AP, et al., 2019).

Entretanto, osteorradionecrose e osteomielite devem ser descartadas por apresentarem sintomas e achados radiográficos semelhantes (DUARTE LFM, et al., 2015). Os tipos de tratamentos para a osteonecrose dos maxilares associada aos bisfosfonatos têm sido estudadas, sendo divididas em manejos conservadores que incluem a adequação do meio bucal, eliminação de doença periodontais, uso de terapia antibiótica sistêmica e manejos invasivos, como a sequestrectomia óssea (CARVALHO LNV, et al., 2018).

Dessa forma, o presente artigo tem como objetivo relatar um caso clínico de um paciente sob o uso crônico de Alendronato de sódio e a ocorrência da osteonecrose em região da maxila, bem como a conduta adotada e tratamento realizado.

\section{DETALHAMENTO DO CASO}

Paciente do sexo feminino, 67 anos, feoderma, foi encaminhada ao serviço de Estomatologia. Na anamnese, afirma ser portadora de diabetes e hipertensa não compensada. Na história médica pregressa, relatou ter realizado tratamento para osteoporose com Alendronato de sódio via oral durante 4 anos, porém está em drug holiday por aproximadamente 5 anos sob orientação médica. Todos os dados foram confirmados por busca ativa de prontuário médico em Unidade de Saúde da Família. Na história odontológica pregressa relata exodontia de dentes incisivos superiores há aproximadamente 8 meses atrás e aparecimento de osso em região anterior de maxila que iniciou há 6 meses, sem sintomatologia.

Ao exame clínico intrabucal, observou-se presença apenas de dente 13 e ausências dentárias na mandíbula. Em região de rebordo alveolar anterior de maxila notava-se extensa exposição óssea com áreas necróticas, acometendo aproximadamente região do dente 14 ao dente 26 (Figura 1).

Figura 1 - Imagem intrabucal apresentando extensa exposição do osso necrótico

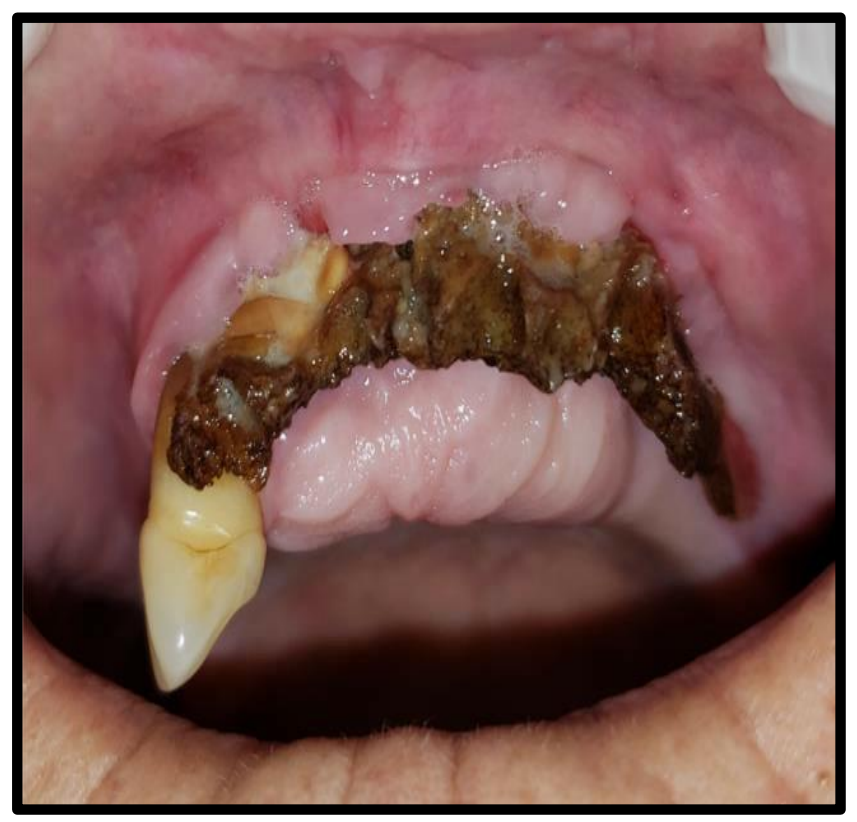

Fonte: Santos WB, et al., 2019. 
Aos exames complementares de radiografia panorâmica e tomografia observou-se presença de reabsorção óssea extensiva, sequestro ósseo por toda extensão da maxila e velamento parcial dos seios maxilares de ambos os lados. Nota-se também aspecto de elemento supranumerário, retido, em posição transversal, apresentando anomalia de forma e tamanho, localizado na região dos dentes 11 e 21 , apresentando reabsorção radicular externa (Figuras 2 e 3).

Figura 2 - Radiografia panorâmica evidenciando manchas radiolúcidas difusas.

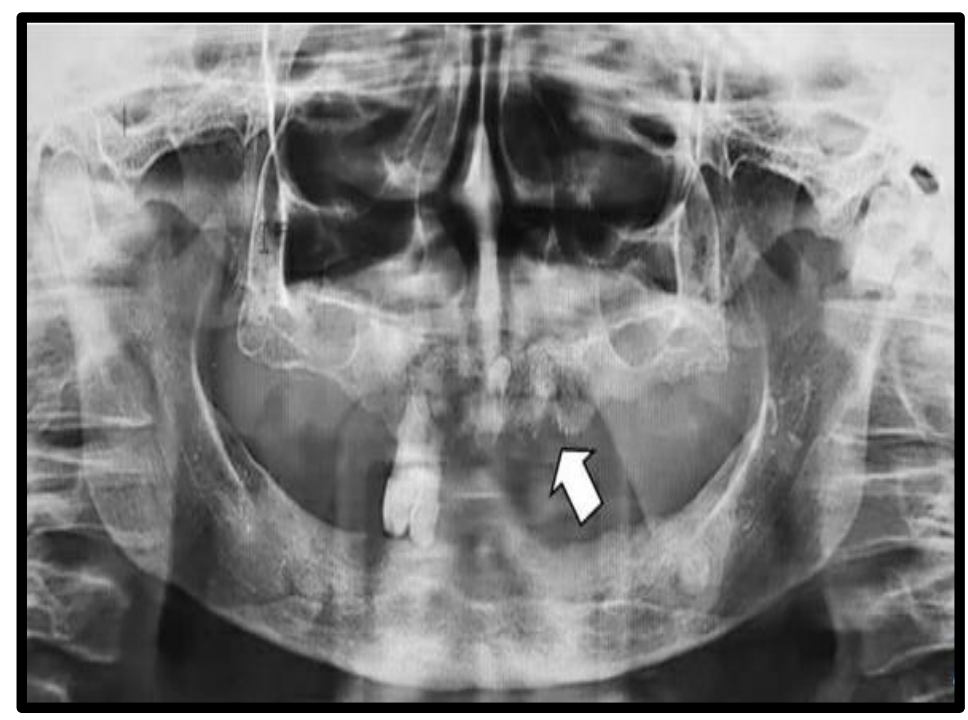

Fonte: Santos WB, et al.,2019.

Figura 3 - Panorâmica tomográfica - Reconstrução axial evidenciando imagem do sequestro ósseo em região anterior de maxilar.

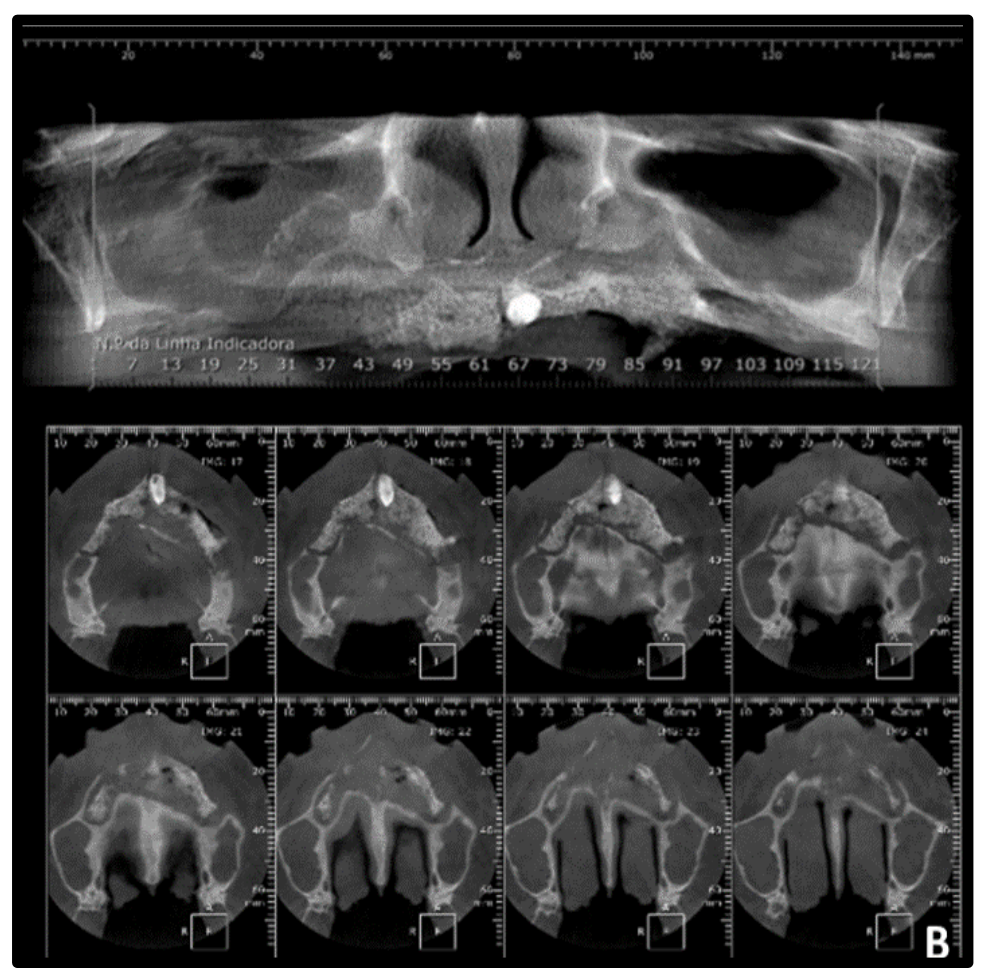

Fonte: Santos WB, et al., 2019 
Como conduta inicial, foi proposto tratamento conservador com bochechos diários com Clorexidina $0,12 \%$ com acompanhamento de 5 meses, pois a paciente não relatou sintomatologia e o quadro clínico apresentava apenas exposição óssea, sem áreas de sequestro ósseo. Após cinco meses osso encontrou-se sequestrado, sendo então feita uma avaliação e planejamento cirúrgico, sendo o tratamento cirúrgico realizado através de sequestrectomia em dois tempos cirúrgicos (Figuras 4 e 5).

Figura 4 - A) Sequestro ósseo removido. B) Pós-operatório imediato com sutura realizada.

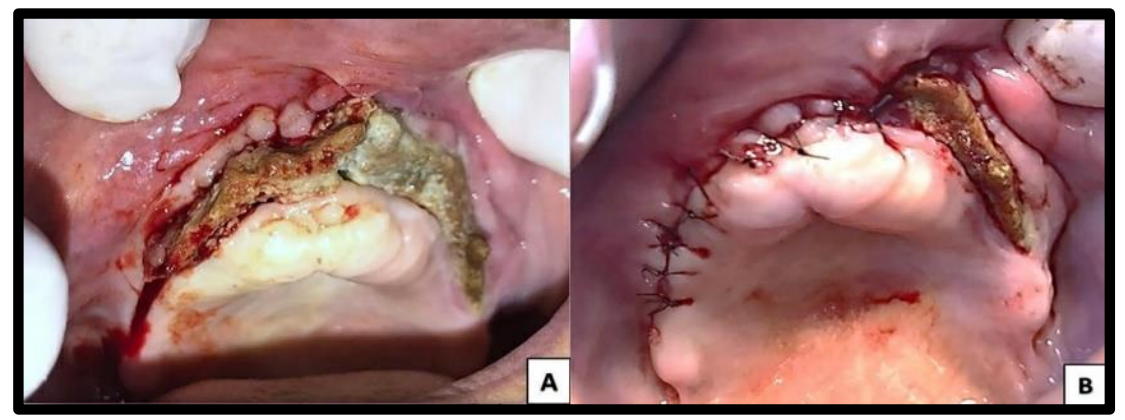

Fonte: Santos WB, et al., 2019.

Figura 5 - A) Pós-operatório de 7 dias. B) Pós-operatório imediato de sequestrectomia à direita.

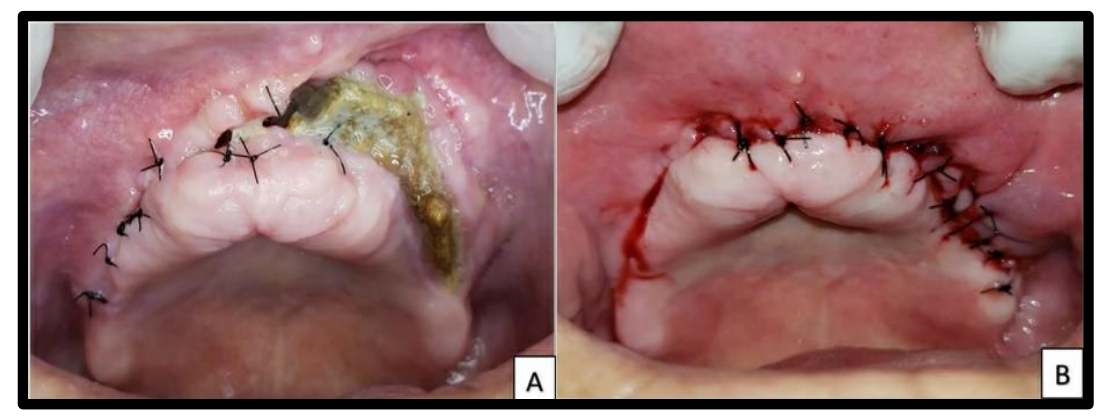

Fonte: Santos WB, et al., 2019.

Os fragmentos ósseos foram submetidos à análise histopatológica, que evidenciaram fragmentos de tecido osteoide trabecular com focos de necrose multifocal. A paciente obteve acompanhamento periódico de 7 dias, 1 mês, 2 meses, 3 meses e 6 meses, para evitar posteriores complicações e possíveis recidivas da lesão, evoluindo sem intercorrências e sem novas exposições ósseas (Figura 6).

Figura 6 - Aspecto intraoral do pós-operatório de 6 meses evidenciando boa cicatrização.

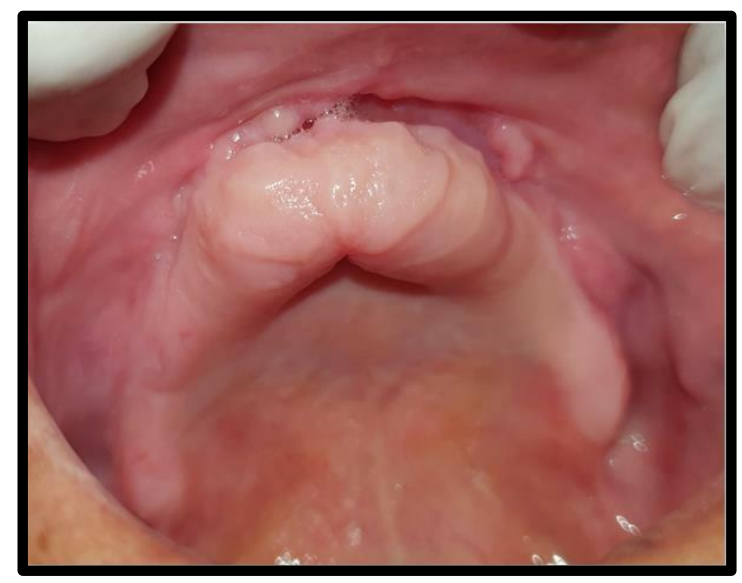

Fonte: Santos WB, et al., 2019. 


\section{DISCUSSÃO}

A osteonecrose dos maxilares é uma doença que afeta tanto a maxila quanto a mandíbula, devido à redução da vascularização, no qual o tecido ósseo perde a capacidade de remodelação, pela deficiência na angiogênese (SOUSA AS, et al., 2018; DUARTE LFM, et al., 2015). Alguns fatores de risco podem estar associados ao desenvolvimento da osteonecrose dos maxilares, dentre os quais podemos citar a potência de cada tipo de bifosfonatos, o tipo de administração, a duração do tratamento, diabetes, uso de álcool e tabaco, presença ou não de traumas cirúrgicos, extrações e higiene oral deficiente (DUQUE MGB, et al., 2017).

Ultimamente, vem sendo constatado uma forte relação no que diz respeito à utilização crônica de bifosfonatos e a osteonecrose dos maxilares. Esta doença é caracterizada pela incapacidade do tecido ósseo afetado em reparar e se remodelar frente a quadros inflamatórios provocados por estresse mecânico (mastigação), exodontias, irritações por próteses ou infecção dentária. Isso é ocasionado em virtude da microbiota presente na cavidade bucal, assim como da sua exposição frequente ao ambiente externo resultantes dos procedimentos dentários, tornando-os, mais sujeitos a infecções (ZANATA A, et al., 2014).

Conforme dados obtidos pelo Food and Drug Administration (FDA) (1999), estima-se que cerca de 5.1 milhões de pacientes acima de 55 anos receberam prescrição de Alendronato no período de 1 ano ao redor do mundo, para controle e tratamento de osteoporose (SOUSA AS, et al., 2018). Diante disto, torna-se clara a preocupação crescente sobre a osteonecrose dos maxilares associada aos bifosfonatos.

De acordo com Jesus AP, et al. (2019), a ocorrência da osteonecrose associada ao uso desses medicamentos estão diretamente relacionadas com a dose, via de administração e a duração do tratamento, sendo de maior incidência em pacientes que fazem o uso administrado por via intravenosa, com aplicação mensal, por um período maior que três anos. Entretanto, pacientes portadores de osteoporose com uso crônico de bisfosfonatos por via oral podem apresentar o desenvolvimento dessa lesão (JESUS AP, et al., 2018). No caso relatado, havia histórico do uso crônico de Alendronato de sódio, por via oral, há 4 anos a fim de prevenir a evolução de um quadro de osteoporose, no qual estima-se que sua incidência seja em torno de $0,1 \%$, podendo aumentar para $0,21 \%$ quando o tratamento se estende por mais de 4 anos.

O alendronato de sódio, classificado como bifosfonato nitrogenado, atua na sinalização de proteínas chaves e interferindo na via do mevalonato. A princípio, por possuir afinidade se fixa na matriz óssea, aproximadamente $50 \%$ da sua dose no processo de absorção permanece no osso e como possui uma meia vida por volta de 10 anos, sua concentração plasmática é reduzida lentamente (COSTA ICS, et al., 2019; MORAIS EF, et al.,2016). A paciente de que se trata, fez o uso por via oral do mesmo que apesar de ser um fármaco com capacidade de reabsorção óssea, apresenta-se com menor potência que o zolendronato (Zometa®), visto que o mesmo é grandemente absorvido por ser administrado por via parenteral, comparado àqueles administrados por via oral.

A Associação Americana de Cirurgiões Orais e Maxilofaciais (AAOMS) ressalta que a osteonecrose dos maxilares causada pelo uso de bifosfonatos pode ocorrer em ambos os sexos, entretanto, há predileção pelo gênero feminino, já que o uso de bifosfonatos é amplamente indicado para tratamento de doenças como câncer de mama e osteoporose que acometem predominantemente mulheres (COLÉTE JZ, et al., 2019). O caso apresentado está em concordância com a literatura no que diz respeito à predileção pelo gênero feminino.

A patogênese da osteonecrose associada ao uso de bifosfonatos ainda não foi completamente esclarecido, porém as hipóteses mais evidentes é a ação direta nos osteoclastos e consequentemente inibição da remodelação óssea levando a hipóxia e posteriormente a necrose do tecido. O aspecto clínico torna-se variado, apresentando áreas expostas de osso necrótico, podendo ou não apresentar fístula e secreção purulenta (LACERDA JP, et al., 2019; VIANA MVG, et al., 2019). Desse modo, a paciente apresentou áreas necróticas do osso, exposto em maxila sem a presença de fístulas.

Em relação à sintomatologia, a osteonecrose pode se manter assintomática a depender do período de tempo, por semanas, meses, inclusive até anos, mas no momento em que surgem sinais e sintomas clínicos, 
estes podem estar associados com dor, mobilidade dentária, edema, eritema e ulceração (DUQUE MGB, et al., 2017). A paciente do presente estudo não apresentava sintomatologia dolorosa.

O diagnóstico de osteonecrose maxilar associada ao uso de bifosfonatos é estabelecido com base em algumas características, tais como o tratamento atual ou anterior utilizando bifosfonatos, exposição óssea na região maxilofacial que persistiu por mais de 8 semanas e sem história de tratamento radioterápico para os maxilares (BROZOSKI MA, et al., 2012).

Segundo Zanata A, et al. (2014), a presença de comorbidades como a diabetes, obesidade, baixa concentração de hemoglobina e diálise renal também estão sendo considerados fatores de risco para 0 desenvolvimento da osteonecrose dos maxilares em pacientes que fazem uso de bifosfonatos. Em concordância com os estudos de Zanata A, et al. (2014); a paciente do presente caso apresentava como fator de comorbidades diabetes e era hipertensa não compensada, sendo estes um dos possíveis fatores de risco ao desenvolvimento da osteonecrose dos maxilares associada ao uso crônico do Alendronato de sódio.

Devido à osteonecrose dos maxilares associada ao uso de bisfosfonatos ser uma doença pouco conhecida, ainda não dispõe de um tratamento fundamentado em orientações padronizadas. O protocolo é conduzido para cada caso, a depender do grau clínico da doença. Sendo assim, tem sido descrito como tratamento ressecções ósseas, desbridamentos, antibioticoterapia, oxigenação hiperbárica, irrigação local com solução antimicrobiana, emprego de plasma rico em plaquetas e laserterapia (ZANATA A, et al., 2014). No caso exposto, como de início a paciente não relatava sintomatologia nem sequestro ósseo, foi proposto um tratamento conservador com bochechos diários com clorexidina 0,12 \% com acompanhamento mensal. Após 5 meses, o aspecto ósseo maxilar da região encontrou-se sequestrado, sendo o tratamento realizado em dois tempos cirúrgicos através da sequestrectomia.

É importância um entendimento detalhado em relação à frequência e as possíveis complicações referentes ao uso de bifosfonatos. Dessa forma, é ideal a compreensão a cerca da utilização desse medicamento para que os profissionais aconselhem adequadamente seus pacientes sobre a relação de risco e benefício durante a realização de procedimentos. Antes de iniciar o tratamento com bifosfonatos o paciente deve passar por um exame da cavidade oral completo, procedimentos invasivos devem ser concluídos e focos infecciosos devem ser excluídos. Essa decisão deve ser feita conjunto com o médico do paciente e o cirurgião-dentista, constituindo assim, uma abordagem de prevenção à osteonecrose (JESUS AP, et al., 2019).

A osteonecrose dos maxilares é um preocupante efeito adverso ocasionado pelo uso crônico de bifosfonatos. É importante que todo paciente que for submeter à terapia com bifosfonatos sejam orientados quanto a prevenção da doença, já que há uma importância considerável em estudos nesta área, visando estabelecer o correto diagnóstico e orientações claras quanto ao mecanismo de ação dos bisfosfonatos. Dessa forma, a avaliação pelo Cirurgião-dentista tem como objetivo a adequação do meio bucal, através do planejamento do protocolo de tratamento, tornando-se válido a interação com o médico do paciente a fim de orientar a importância da manutenção da saúde bucal para prevenção de possíveis complicações da doença.

\section{REFERÊNCIAS}

1. BROZOSKI MA, et al. Osteonecrose maxilar associada ao uso de bisfosfonatos. Revista Brasileira de Reumatologia, 2012; 52(2): 265-270.

2. CARVALHO LNV, et al. Osteonecrose dos maxilares relacionada ao uso de medicações: Diagnóstico, tratamento e prevenção. CES Odontología, 2018; 31(2): 49-63.

3. COLÉTE JZ, et al. Implantes em pacientes com osteonecrose dos maxilares associado ao uso de bifosfonatos. Relato de caso e revisão de literatura. Arch Health Invest, 2019; 8(1): 20-27.

4. COSTA ICS, et al. RELAÇÃO DO USO DOS BIFOSFONATOS COM A OSTEONECROSE DOS MAXILARES: RELATO DE CASO. Odontol. Clín. Cient., 2019; 18(2): 143- 146.

5. DIAS DHS, et al. Osteonecrose associada ao uso de alendronato de sódio: relato de caso. Rev Higei@. UNIMES, 2016.

6. DUARTE LFM, et al. Surgical Treatment of Bisphosphonate-Related Osteonecrosis of the Jaws with the Use of Buccal Fat Pad: Case Report. Braz Dent J, 2015; 26(3):317-320.

REAS/EJCH | Vol.12(2) | e2398 | DOI: https://doi.org/10.25248/reas.e2398.2020 Página 7 de 8 
7. DUQUE MGB, et al. Osteonecrose mandibular associada ao uso de bisfosfonatos tratada com plasma rico em fibrina leucocitária: relato de caso. RFO, 2017; 22(3): 368-373.

8. FILGUEIRA SL, et al. Manifestações clínicas da osteonecrose induzida por medicamentos. Rev Ciência atual, 2019; 13(1): 02-13.

9. JESUS AP, et al. Tratamento cirúrgico para osteonecrose dos maxilares induzida por bisfosfonatos: relatos de casos. RFO UPF, 2019; 24(1): 22-30.

10. LACERDA JP. OSTEONECROSIS OF THE MAXILARIES ASSOCIATED WITH USE OF BIPHOSPHONATE. BJIHS, 2019; 1 (2): 1-7.

11. LIMA BKS, et al. Perfil microbiológico bucal dos pacientes portadores de osteonecrose maxilar induzida por bisfosfonatos. Rev. Investig, Bioméd, 2017; 9(2): 187-97

12. MORAIS EF, et al. OSTEONECROSE DOS MAXILARES ASSOCIADO AO USO DE ALENDRONATO ORAL EM PACIENTE SOB TRATAMENTO DE OSTEOPOROSE, 2016; 5(1): 21-29.

13. SOUSA AS, et al. Protocolo de atendimento odontológico de pacientes em tratamento com bisfosfonatos. Rev Saúde, 2018; 12(1-2): 54-61.

14. VIANA MVG, et al. Considerações clinicas sobre o uso do L-PRF na terapêutica de osteonecrose medicamentosa dos maxilares: relato de caso. Braz. J. Healthy. Rev., 2019; 2(4): 3318-3327.

15. ZANATA A, et al. Osteonecrose mandibular associada ao uso de bisfosfonato de sódio em paciente com mieloma múltiplo. Rev Port Estomatol Med Dent Cir Maxilofac, 2014; 55(2):115-120. 